\title{
MODELAGEM DE RECRUTAMENTO EM FLORESTAS
}

\author{
Luiz Marcelo Brum Rossi*, Henrique Soares Koehler**, Julio Eduardo Arce***, \\ Carlos Roberto Sanquetta*** \\ *Eng. Florestal, M.Sc., Embrapa Amazônia Ocidental - mrossi@cpaa.embrapa.br \\ **Eng. Florestal, Dr., Depto. de Fitotecnia e Fitossanitarismo, UFPR - koehler@ufpr.br \\ ***Eng. Florestal, Dr., Depto. de Ciências Florestais, UFPR - jarce@ufpr.br - sanquetta@ufpr.br \\ Recebido para publicação: 28/08/2006 - Aceito para publicação: 07/02/2007
}

\begin{abstract}
Resumo
As florestas são compostas por árvores de muitas espécies, de tamanhos diferentes, com variadas e indeterminadas idades e crescendo sobre distintas condições ambientais. Esse cenário faz com que seja necessária a busca por técnicas adequadas para a modelagem da dinâmica e da produção dessas florestas. A dinâmica das florestas é basicamente formada pelos componentes crescimento, mortalidade e recrutamento. Esses três componentes são fundamentais para a modelagem e subseqüente simulação e projeção do crescimento e da produção da floresta em longo prazo. $\mathrm{O}$ recrutamento é o evento no qual o tamanho (diâmetro ou altura) de uma árvore alcança uma dimensão mínima de medição, ou seja, é o ingresso na menor classe considerada. O objetivo deste trabalho foi apresentar alguns aspectos gerais da modelagem do recrutamento em florestas. A revisão mostra as definições de recrutamento; os aspectos ambientais da ocorrência do evento; as maneiras de obtenção de dados de recrutamento; as taxas obtidas em diferentes tipos florestais; as distintas formas de abordagem da modelagem do componente; as funções, variáveis e tipos de modelos mais comumente utilizados; e exemplos de modelos e sistemas que incluem o recrutamento na modelagem da produção e da dinâmica florestal.

Palavras-chave: Modelos; ingresso; simulação; predição.
\end{abstract}

\begin{abstract}
Forests recruitment modelling. Forests are composed of trees of several species of different sizes, multiple and undetermined ages, growing under distinct environmental conditions. These circumstances make necessary the search for adequate techniques for modelling forest dynamics and yield. Forest dynamics are basically formed by three components: recruitment, growth, and mortality. These components are essentials for modelling the forthcoming steps of simulation, growth and yield prediction. Recruitment may be defined as the event on which the tree size (diameter or height) reaches determined value during measurements intervals. The main objective of this paper is to present the general guidelines for modelling forests recruitments. The literature review presents the definitions for recruitment, environmental traits of the event occurrence, the way recruitment data are obtained, the rates of recruitment under different forests situations, the most common modelling approaches for recruitment, the functions, variables and models types most commonly used and some examples of systems and models, which include recruitment, aiming at modelling forest yield and dynamics.
\end{abstract}

Keywords: Models; ingrowth; simulation; prediction.

\section{INTRODUÇÃO}

As comunidades florestais são dinâmicas e as mudanças ocorrem continuamente em níveis de população, de espécies e de indivíduos ao longo do tempo, mesmo que a comunidade como um todo seja estável, devido ao equilíbrio entre crescimento, recrutamento e mortalidade (FELFILI, 1995). De acordo com Lewis et al. (2004), as estimativas de mortalidade e recrutamento são descritores fundamentais das populações de árvores em florestas. Comparações entre estudos são importantes tanto para entender a dinâmica das florestas quanto para fazer generalizações sobre o padrão no tempo e espaço e para inferir sobre as causas fundamentais da dinâmica. 
Os inventários em parcelas permanentes podem ser usados para descrever a dinâmica da população de árvores, isto é, para determinar taxas de recrutamento e mortalidade. A obtenção de estimativas de recrutamento em um tamanho mínimo de indivíduo é mais difícil do que estimar mortalidade de árvores, visto que o número de plantas recrutadas irá variar largamente dependendo do tamanho do intervalo de tempo do inventário e do tamanho da parcela (KOHYAMA; TAKADA, 1998).

De acordo com Clark et al. (1999), existem duas visões relativas ao papel do recrutamento na dinâmica florestal. A primeira é que as populações possuem recrutamento limitado, com baixa e incerta produção de sementes ou estabelecimento de plântulas, estando essas entre as causas para a ausência ou raridade. A falta de fontes locais de sementes ou pouca produção de sementes combinada com a dispersão restrita tem impactos permanentes na dinâmica da população. A segunda visão atribui um papel mais limitado na dinâmica da floresta ao suprimento de sementes e estabelecimento das plântulas. Aqui o foco muda para distribuições e qualidade dos microssítios e fatores que afetam o crescimento e a mortalidade no banco de sementes e nos estágios de plântulas.

Muhairwe (2003) afirma que o desenvolvimento de modelos de crescimento para florestas de espécies mistas ineqüiâneas deve estar voltado para as mudanças na estrutura e composição da floresta e refletir as respostas das operações silviculturais. Vanclay (1994b) identifica os componentes de tais modelos, como crescimento, ingresso (regeneração e recrutamento), mortalidade (natural e catastrófica) e colheita. Com os sistemas de extração seletiva praticados nas florestas, o ingresso é um componente essencial para o manejo em longo prazo e para a previsão da produção. Carvalho (1997) também afirma que recrutamento, mortalidade e incremento diamétrico estão entre os poucos instrumentos para se fazer predições adequadas sobre a produção futura de um povoamento florestal.

Muitos modelos de crescimento podem desconsiderar a simulação da regeneração por causa da falta de dados ou de dificuldades na modelagem, ou então porque é desnecessário, pelo fato de que a silvicultura envolve derrubada e replantio. Entretanto, a regeneração natural forma um componente essencial de seleção dos sistemas de colheita usados no manejo de florestas, e o recrutamento pode, então, contribuir substancialmente para a área basal do futuro povoamento e assim influenciar o crescimento e a previsão de produção em longo prazo (VANCLAY, 1992, 1994b).

Na modelagem de povoamentos eqüiâneos, a entrada de plântulas ou ingresso pode ser ignorada. Já na modelagem de um povoamento ineqüiâneo, o ingresso deve ser considerado cuidadosamente por causa da teoria da população, que claramente mostra a importância da influência do comportamento da regeneração no desenvolvimento, estrutura e estabilidade de populações, e porque o ingresso é um componente importante do crescimento do povoamento (LEAK; GRABER, 1976).

\section{Definição de recrutamento}

Carvalho (1997) define recrutamento como a admissão de um ser em uma determinada população ou comunidade. O recrutamento de plântulas pode ser confundido com o seu aparecimento ou germinação. Muitas vezes o recrutamento também é chamado de ingresso, e nesse caso deve ser definido como o processo pelo qual as árvores pequenas aparecem em um povoamento, como, por exemplo, em uma parcela permanente, após a primeira medição.

O recrutamento é usualmente quantificado por meio do número de árvores ou plântulas que alcançam ou excedem um tamanho limite específico em certo período, como resultado de diferentes processos de regeneração, como o estabelecimento, crescimento e mortalidade de plântulas (LEXERøD; EID, 2005).

Segundo Kohyama e Takada (1998), obtêm-se taxas de recrutamento aparente simplesmente pela contagem de árvores que são menores que um tamanho mínimo em um inventário, mas que entraram nesse tamanho em um segundo inventário. Moser (1972) define ingresso como o acréscimo de árvores acima de um limite de tamanho arbitrariamente definido, representando um aumento das árvores contáveis que estão competindo por um nicho no povoamento mensurável.

Vanclay (1994b) diferencia os termos regeneração e recrutamento, sendo que regeneração é a renovação de povoamentos florestais por meios naturais ou meios artificiais (semeadura e plantio), enquanto que o recrutamento refere-se aos indivíduos que alcançaram um tamanho especificado (em altura ou em diâmetro). Os conceitos estão relacionados, e a distinção entre eles depende de eventos arbitrários no desenvolvimento de um indivíduo, desde a semente até uma pequena árvore. 
Para Swaine et al. (1987), a taxa de recrutamento de uma espécie é a manifestação da fecundidade, crescimento e sobrevivência de plantas juvenis dessa espécie na população. Porté e Bartelink (2002), em uma revisão de modelos para florestas, consideram que a regeneração é definida como a progenia estabelecida de uma matriz, ou as plântulas ou mudas existentes em um povoamento. O sinônimo recrutamento foi principalmente usado na literatura nos modelos de distribuições (modelos SIRENA e CAFOGROM) e modelos de árvores dependentes da distância (SPACE, SORTIE, FORMOSAIC, TROLL). O estabelecimento é o resultado do processo de regeneração. Esse termo foi somente algumas vezes usado sozinho (ARCADIA) ou juntamente com a palavra recrutamento (FOREST, TROLL) ou como regeneração somente (FORSKA, OUTENIQUA, ZELIG, KOPIDE).

$\mathrm{O}$ ingresso corresponde à quantidade de árvores (em número, volume ou área basal) que são menores que um determinado diâmetro mínimo, ou o limite de altura no início de um período de crescimento, e que durante esse período atingiram o tamanho prescrito. $O$ ingresso é freqüentemente restrito quando se refere à regeneração, mas somente empregado em modelos de distribuição baseados em matrizes como FOREST, FIBER 3.0 e no modelo de Hann (1980). Conseqüentemente, o termo recrutamento parece mais adequado para referir-se ao processo de instalação de novas plântulas no povoamento (PORTÉ; BARTELINK, 2002).

\section{Abordagem da modelagem de recrutamento}

A predição do ingresso pode ser abordada em três estágios: 1) modelagem da produção de sementes e germinação; 2) modelagem da regeneração que tenta predizer o desenvolvimento das árvores a partir das sementes ou plântulas; 3) modelagem do recrutamento, a qual prediz o número de árvores que atinge um tamanho predeterminado (MUHAIRWE, 2003).

De acordo com Vanclay (1992), como os dados adequados para modelagem da regeneração são difíceis de obter, muitos modelos predizem o recrutamento em vez da regeneração. Esses modelos predizem o número de árvores que alcançam ou que excedem o limite de algum tamanho nominal especificado (por exemplo: $3 \mathrm{~m}$ de altura, $10 \mathrm{~cm}$ de diâmetro).

Vanclay (1994a) distingue dois procedimentos para a modelagem do recrutamento: o estático e o dinâmico. O procedimento estático leva relativamente pouco em conta as condições do povoamento e assim prediz uma quantidade constante de recrutamento, indicando uma média esperada em longo prazo sob condições típicas. O procedimento dinâmico corresponde às condições do povoamento, predizendo o recrutamento como uma função da densidade do povoamento, da composição e de outros parâmetros.

A abordagem estática da predição do recrutamento considera que a quantidade de recrutamento observado durante o período de coleta de dados reflete a média em longo prazo, e que essa quantidade não irá variar grandemente entre os períodos de projeção preditos pelo modelo. Tais suposições do recrutamento estático são comuns em projeção da tabela de povoamentos e em métodos matriciais. Procedimentos matriciais realísticos podem predizer a diminuição do recrutamento com o aumento da densidade do povoamento ou somente com a morte de outra árvore. Embora essas abordagens sejam um tanto empíricas, elas podem fornecer estimativas úteis de recrutamento para povoamentos que não diferem grandemente dos povoamentos-fontes usados para o desenvolvimento do modelo (VANCLAY, 1992).

Quando os dados permitem, o melhor procedimento é estimar o recrutamento como uma função do sítio e das condições do povoamento. Diversos desses modelos têm sido publicados, e variam de altamente empíricos até aqueles com uma base biológica. As variáveis comumente usadas incluem a produtividade do sítio, a densidade do povoamento (área basal do povoamento, número de árvores, índices de competição) e tempo desde a última colheita. A inclusão de detalhes da última exploração no modelo pode ajudar a obter um bom ajuste aos dados, porém isso pode limitar as aplicações do modelo, já que esses detalhes podem não ser conhecidos para todos os povoamentos. Quando diversas espécies estão envolvidas, os modelos podem predizer o recrutamento para cada grupo de espécies independentemente, ou podem predizer o total e então determiná-los às espécies candidatas (VANCLAY, 1994b).

Alguns modelos usam estimativas da densidade de arvoretas (número de plantas em classes diamétricas) nas equações, considerando que essas estimativas permanecerão constantes no tempo, e que assim que as arvoretas são recrutadas, nova regeneração toma seu lugar. Entretanto, isso limita a utilidade dos modelos na extrapolação de dados de inventário, já que tais dados (densidade de arvoretas) podem não ser registrados durante o inventário operacional (VANCLAY, 1992). 
De acordo com Vanclay (1989), a natureza irregular do recrutamento sugere o uso de uma função estocástica, porém a predição eficiente da produção requer um modelo determinístico. A fimm de assegurar estimativas realistas de recrutamento, é apropriado usar uma função determinística para predizer o recrutamento total e a divisão dele entre os grupos de espécies. Procedimentos mais sofisticados, como os de Botkin et al. (1972), só são possíveis se a informação espacial estiver disponível e se um procedimento estocástico for aceitável. Entretanto, as necessidades da eficiente predição da produção, utilizando dados existentes de inventários, muitas vezes excluem essas metodologias.

\section{Funções para modelagem de recrutamento}

Hann (1980) fez a predição do recrutamento para Pinus ponderosa empregando uma equação exponencial em função do índice de sítio, área basal do povoamento e área basal na menor classe de tamanho, simulando ciclos de cinco anos. Duas equações foram usadas para modelar o ingresso ineqüiâneo. A primeira prediz o número total de árvores que ingressam e a segunda prediz a proporção de árvores que irá crescer e passar da classe de $10 \mathrm{~cm}$ de DAP para a classe de 12,5 cm de DAP durante o período de crescimento. Após examinar previamente informações silviculturais e de medições, foi considerado que os fatores que possivelmente influenciam a taxa de ingresso para povoamentos ineqüiâneos incluíam o potencial do povoamento em produzir cones, a estrutura e nível de competição, índice de sítio, diâmetro médio do povoamento e média quadrática do diâmetro do povoamento. Com base nesses fatores, foram definidas variáveis independentes apropriadas. O modelo final de ingresso total (IT) foi:

$$
I T=b_{0} S\left(A B C L_{1}\right)^{b_{1}}\left(1,0+A B C L_{2}\right)^{b_{2}} e^{\left(b_{3} A B^{b_{4}}+b_{5} A_{1} A B^{b_{4}}\right)}
$$

onde: $S$ é o índice de sítio; $A B$ é a área basal total; $A B C L_{1}$ é a área basal na classe de DAP de 10 a 12,5 $\mathrm{cm} ; A B C L_{2}$ é a área basal nas classes de DAP igual ou maiores que $17,5 \mathrm{~cm}$ e $A$ é a transformação sigmoidal do período de crescimento de cinco anos.

Lynch e Moser (1986) estudaram o ingresso de folhosas e coníferas em povoamentos mistos. O ingresso dessas espécies foi influenciado pelo tamanho da árvore e pela densidade. Medidas de tamanho da árvore e densidade obtidas dos dados disponíveis da parcela incluem o número de árvores, área basal e diâmetro médio. As seguintes equações foram usadas para predição da taxa de ingresso (número de árvores) para os dois grupos de espécies, coníferas (2) e folhosas (3):

$$
\begin{aligned}
& d N_{1} / d t=b_{1}\left(N_{1}\right)^{b_{2}} \exp \left(b_{3} B_{1}+b_{4} B_{2}\right) \\
& d N_{2} / d t=b_{0} \exp \left[b_{1}\left(\frac{S_{1}}{N_{1}}\right)+b_{2}\left(\frac{S_{2}}{N_{2}}\right)\right]
\end{aligned}
$$

onde: $B_{k}$ é a área basal do grupo de espécies $k ; S_{k}$ é a soma de diâmetros do $k ; N_{k}$ é o número de árvores do grupo $k$; sendo $k=1$ para coníferas e $k=2$ para folhosas. A equação (3), para folhosas, é baseada na função não-linear da área basal média por árvore usada por Moser (1972) para predizer a taxa de mudança do ingresso de árvores em povoamento misto de folhosas. O diâmetro médio para cada grupo de espécies foi usado na equação para folhosas (3) em vez da área basal média para todas as espécies, como usado por Moser (1972). Os sinais dos coeficientes na equação de folhosas indicaram que o tamanho médio da árvore tende a ser inversamente relacionado ao ingresso para esses dados.

Vanclay (1989) fez a predição do recrutamento total no DAP de $20 \mathrm{~cm}$, nas florestas pluviais do norte do estado de Queensland (Austrália), em função da área basal do povoamento e qualidade do sítio:

$$
N r=b_{0}+b_{1} S B A+b_{2} S Q
$$


onde: $N r$ é o número de árvores recrutadas (árvores ha $\left.{ }^{-1} \mathrm{ano}^{-1}\right) ; S B A$ é a área basal $\left(\mathrm{m}^{2} \mathrm{ha}^{-1}\right) \mathrm{do}$ povoamento e $S Q$ é a qualidade do sítio. A composição desse recrutamento foi determinada pela predição da proporção em cada um dos cinco grupos de espécies. A proporção para cada grupo de espécies foi predita a partir da área basal do povoamento, qualidade do sítio e área basal do grupo:

$$
P_{i}=\left(1+\exp \left[b_{0}+b_{1} S B A+b_{2} B_{i}+b_{3} B_{i} S Q\right]\right)^{-1}
$$

onde $B_{i}$ é área basal das espécies do grupo $i\left(\mathrm{~m}^{2} \mathrm{ha}^{-1}\right)$. Essas proporções foram então padronizadas para assegurar soma igual a um, na forma: $P_{i}^{\prime}=P_{i} / \Sigma P$. Apesar de mais simplista, esse modelo permite suficiente flexibilidade para grupos dinâmicos de espécies e fornece resultados razoáveis.

A probabilidade de que qualquer recrutamento ocorra foi predita separadamente para cada espécie, ao contrário do procedimento usado no modelo PROGNOSIS, no qual a probabilidade coletiva de regeneração é modelada. Cinco grupos de espécies são usados no modelo NORM por Vanclay (1992, 1994a) para estimar a probabilidade anual de recrutamento a partir da área basal do povoamento, a presença da espécie no povoamento e o número de anos desde o último distúrbio. A função usada foi:

$$
\mathrm{P}_{\mathrm{r}, \mathrm{i}}=\left\{1+\exp \left(\mathrm{b}_{0}+b_{1} Z_{i}+b_{2} G+b_{3} \operatorname{Ln} G+b_{4} S_{v}+b_{5} t_{s} e^{b_{6} t_{s}}\right)\right\}-1
$$

onde: $Z_{i}$ é uma variável binária indicando a presença da espécie $i$ no povoamento $(1=$ presença e $0=$ ausência); $G$ é a área basal do povoamento $\left(\mathrm{m}^{2} \mathrm{ha}^{-1}\right) ; t$ é o número de anos desde o tratamento silvicultural; e $S_{v}$ é uma variável binária relacionada ao tipo de solo. Essa função pode ser empregada de uma maneira estocástica ou determinística. A implementação determinística soma as probabilidades anuais para cada espécie até que a probabilidade exceda a unidade.

No modelo de Vanclay (1994a) para a função final de recrutamento, as espécies foram agrupadas para analisar a quantidade de regeneração, e oito grupos de espécies foram usados para estimar a função final. O recrutamento foi predito a partir da área basal do povoamento, índice de produtividade do sítio e abundância relativa das espécies no povoamento, na forma:

$$
L n N_{r, i}=b_{0}+b_{1} G+b_{2} \operatorname{Ln}\left(\frac{N_{i}}{N}+0,2\right)+b_{3} S_{\Delta d}+b_{4} S_{a}
$$

onde: $N_{i}$ é o número de árvores da espécie $i$ no povoamento; $S_{\Delta d}$ é o índice de produtividade do sítio; $S_{a}$ é uma variável binária do tipo de solo.

Nos estudos de simulação em floresta tropical pluvial na Austrália, Vanclay (1994a) modelou a probabilidade de recrutamento como:

$$
P=\left(1+\exp \left[b_{0}+b_{1} P R E S+b_{2} A B+b_{3} \log A B+b_{4} T R+b_{5} S O L O\right]\right)^{-1}
$$

onde: $P$ é a probabilidade anual de ocorrência do recrutamento; $A B$ é a área basal $\left(\mathrm{m}^{2} \mathrm{ha}^{-1}\right)$ do povoamento das árvores maiores que $10 \mathrm{~cm}$ de DAP; PRES é uma variável binomial que tem o valor 1 se a espécie está presente no povoamento existente (maior que $10 \mathrm{~cm}$ ) e 0 de outro modo; TR é a resposta do tratamento $\left(T R=t e^{t / 9}\right.$, onde $t$ é o número de anos desde o último tratamento silvicultural); e $S O L O$ é o tipo de solo. A quantidade de recrutamento foi predita como:

$$
\log N=b_{0}+b_{1} \log A B+b_{2} \log (R N O+0,2)+b_{3} S Q+b_{4} S O L O
$$

onde: $N$ é o número esperado de árvores recrutadas (árvores $\mathrm{ha}^{-1} \mathrm{ano}^{-1}$ ); $A B$ é a área basal do povoamento; $R N O$ é o número relativo de árvores ( $>10 \mathrm{~cm}$ DAP) das espécies dentro da parcela; $S Q$ é a qualidade de sítio usando um índice de crescimento; e $S O L O$ é o tipo de solo.

Kohyama (1992) utilizou duas funções para a obtenção da taxa de recrutamento $R_{i}\left(t, x_{0}\right)$ de plantas a partir do banco de plântulas a um valor mínimo de DAP $x_{0}$ no tempo $t$. O primeiro modelo considera que o suprimento de plântulas é independente da densidade de árvores matrizes na parcela de 
simulação, e que a sobrevivência das plântulas e/ou recrutamento para a classe de tamanho maior ou igual ao tamanho $x_{0}$ é suprimida pelo efeito de "sombreamento":

$$
R_{i}\left(t, x_{0}\right)=d_{i} \exp \left[-d_{1 i} B\left(t, x_{0}\right)\right]
$$

onde: $d_{i}\left(\mathrm{~m}^{2} \mathrm{ano}^{-1}\right)$ é a taxa de recrutamento potencial da espécie $i$ sem efeito de "sombreamento" ou competição e $d_{l i}\left(\mathrm{~m}^{2} \mathrm{~cm}^{-2}\right)$ é o coefíciente de redução devido ao sombreamento na espécie $i$. A variável $B\left(t, x_{0}\right)$ é a área basal cumulativa para árvores $\geq x_{0}$ ou a área basal do povoamento. O outro modelo é semelhante ao anterior, exceto que o potencial de suprimento de plântulas sem "sombreamento" é uma função linear da densidade de árvores matrizes na parcela:

$$
R_{i}\left(t, x_{0}\right)=d^{\prime}{ }_{i} B_{i}\left(t, x_{0}\right) \exp \left[-d^{\prime}{ }_{l i} B\left(t, x_{0}\right)\right]
$$

onde: $d^{\prime}{ }_{i}\left(\mathrm{~cm}^{2}\right.$ ano-1 $)$ é a taxa de recrutamento per capita da espécie $i$ e $d^{\prime}{ }_{1 i}\left(\mathrm{~m}^{2} \mathrm{~cm}^{-2}\right)$ é o coeficiente de redução. Esse modelo considera que $B_{i}\left(t, x_{0}\right)$, ou a área basal do povoamento das árvores das espécies $i \geq$ $\mathrm{x}_{0}$, expressa a densidade de árvores-mães. Embora seja preferível usar a área basal cumulativa acima do tamanho crítico de produção de sementes de cada espécie, foi usado $B_{i}\left(t, x_{0}\right)$ por simplicidade e devido à falta de dados reais sobre o tamanho crítico. O primeiro modelo expressa o processo da sucessão secundária em uma pequena área circundada por floresta com fonte de sementes, enquanto o segundo modelo expressa a dinâmica de um sistema florestal fechado de maior área onde a fonte de sementes é limitada ao sítio de interesse.

Kohyama et al. (2001) aplicaram um simulador da dinâmica em floresta mista de dipterocarpáceas na Indonésia em inventários com três anos de intervalo. Os eventos de recrutamento observados no limite inferior $\left(x_{0}\right)$ de $6 \mathrm{~cm}$ de DAP foram muito pequenos para detectar a dependência da taxa de recrutamento em relação à densidade local, sendo empregada a técnica da estimativa $G f$ (KOHYAMA; TAKADA, 1998). Essa estimativa da taxa de recrutamento é a densidade de árvores na menor classe de tamanho, multiplicada pela taxa média de crescimento dessa classe. A área basal total acima de $6 \mathrm{~cm}$ de DAP $B\left(x_{0}\right)$ foi usada para projetar a dependência em relação à densidade local da estimativa $G f$ de recrutamento $R\left(\mathrm{~m}^{2}\right.$ ano $\left.{ }^{-1}\right)$. Para isso foi utilizado um modelo linear na forma:

$$
R=d\left[1-d_{1} B\left(x_{0}\right)\right]
$$

Os autores encontraram correlação negativa entre taxa de recrutamento e a área basal local.

Para a modelagem das florestas áridas de Uganda, Namaalwa et al. (2005) empregaram a estratégia de modelagem em dois estágios. No primeiro estágio, funções para a probabilidade de recrutamento durante o período do inventário foram desenvolvidas para cada classe da espécie $i$, enquanto que funções condicionais para o número de árvores recrutadas por hectare foram desenvolvidas no segundo estágio com base nas parcelas com recrutamento. No primeiro estágio, o ingresso foi considerado uma variável binária para predição da probabilidade de ingresso. A seguinte função logística foi usada:

$$
P I_{i T}=1 / 1+\exp \left(-\left(b_{i 0}+b_{i 1} X_{2}+b_{i 2} X_{3}\right)\right)
$$

onde: $P I_{i T}$ é a probabilidade de ingresso do grupo de espécie $i$ durante o período de inventário $T ; X_{2}$ é a densidade do povoamento e $X_{3}$ é a área basal das espécies. No segundo passo, o relacionamento entre o número árvores recrutadas por hectare (para o período do inventário) e as variáveis independentes foram expressas com o uso da seguinte função:

$$
\operatorname{Ln} I_{i T}=b_{i 0}+b_{i 1} \operatorname{Ln} X_{2}+b_{i 2} \operatorname{Ln} X_{3}
$$

onde $I_{i T}$ é o número de árvores que ingressaram na menor classe de diâmetro para o grupo de espécies $i$ durante o período do inventário.

Muhairwe (2003) afirma que um modelo pode ser construído para predizer a probabilidade $p$ de não ocorrer ingresso e então obter a probabilidade de ingresso $q$ como $q=1-p$. O modelo logístico 
selecionado para predição da probabilidade de não ocorrer ingresso nas florestas do oeste de Nova Gales do Sul (Austrália) foi:

$$
P(0)=\left[1+\exp \left(-\left(b_{0}+b_{1} A B+b_{2} \operatorname{LnAB}+b_{3} \operatorname{LnSPH}+b_{4}\left(\frac{S D I}{S P H}\right)+b_{5} T R M T\right)\right)\right]^{-1}
$$

onde: $A B$ é a área basal do povoamento $\left(\mathrm{m}^{2} \mathrm{ha}^{-1}\right) ; S P H$ é o estoque do povoamento (árvores ha $\left.{ }^{-1}\right) ; S D I$ é o índice de densidade do povoamento (árvores $\mathrm{ha}^{-1}$ ); TRMT é uma variável dummy para o tratamento silvicultural. A inclusão da área basal e do logaritmo da área basal foi justificada porque asseguram que uma densidade baixa do povoamento fornece probabilidades mais altas de recrutamento do que em povoamentos com alta densidade, o que foi consistente com os dados disponíveis. O índice de densidade do povoamento foi usado, mesmo que altamente correlacionado com a área basal, porque é uma boa medida de estoque em povoamentos multiespecíficos ineqüiâneos. Para o segundo estágio, foi determinado o número de árvores recrutadas. Como o recrutamento depende da condição da parcela, a área basal e o estoque e suas variáveis transformadas foram as variáveis candidatas ao modelo. Entretanto, quando o estoque foi incluído, o modelo tendeu a apresentar predições irreais, e por isso o modelo usou somente a área basal. O modelo selecionado foi ajustado como uma função não-linear:

$$
N=\exp \left[b_{0}+b_{1} \operatorname{Ln}(A B)\right]
$$

Huebschmann et al. (2000) descreveram um sistema de equações para modelagem do crescimento e produção em povoamentos naturais de Pinus echinata, no qual usaram o seguinte modelo:

$$
T_{p, 1}=b_{0}+b_{1} T_{p}-b_{2} B_{s}
$$

onde: $T_{p, 1}$ é o número anual de árvores de $P$. echinata ingressando na classe comercial; $T_{p}$ é o número de árvores da espécie no povoamento; e $B_{s}$ é a área basal de todas as espécies no povoamento. $\mathrm{O}$ ajuste obtido teve um coeficiente de determinação $\left(\mathrm{R}^{2}\right)$ de 0,60 .

$\mathrm{Na}$ simulação da dinâmica de florestas tropicais, Robert (2003) calculou o recrutamento dividindo a área do povoamento em quadrados de $2 \mathrm{~m}$ × $2 \mathrm{~m}$, onde a probabilidade de ter uma árvore recrutada dentro do i-ésimo quadrado foi obtida pela função:

$$
P_{r e c, i, t}=1 /\left(1+e^{b_{0}+b_{1} S B A_{i, t}}\right)
$$

onde $\mathrm{SBA}_{\mathrm{i}, \mathrm{t}}$ é a soma da área basal das árvores localizadas dentro de um círculo de 7,5 $\mathrm{m}$ de raio e centralizado no i-ésimo quadrado.

As tentativas de Spathelf e Durlo (2001) em explicar o ingresso, usando características de uma floresta úmida subtropical no sul do Brasil, não tiveram sucesso. No modelo usado, $i=f(G, N)$, nenhum coeficiente significante foi encontrado.

Para avaliar o crescimento e produção de uma Floresta Semidecídua Montana no estado de Minas Gerais, Scolforo et al. (1996) utilizaram um modelo de recrutamento em função do DAP na forma:

$$
I i=b_{0} \exp \left(b_{1} D_{i}\right)
$$

A função obteve um excelente ajuste com coeficiente de determinação $\left(\mathrm{R}^{2}\right)$ com valor de 0,999 .

No sistema CAFOGROM, um modelo empírico de grupos para manejo de florestas exploradas na Amazônia brasileira, Alder e Silva (2000) usaram a função:

$$
I_{g}=0,019 L_{g}^{1,86}
$$

onde $I_{g}$ é o recrutamento da área basal em $\mathrm{m}^{2} \mathrm{ha}^{-1} \mathrm{ano}^{-1}$ e $L_{g}$ é a área basal perdida na colheita ou desbaste e na mortalidade associada, em $\mathrm{m}^{2} \mathrm{ha}^{-1}$. O coeficiente de determinação $\left(\mathrm{R}^{2}\right)$ encontrado foi de 0,596 para 
os dados combinados de duas regiões (Jari e Tapajós), mostrando a correlação entre o recrutamento e a área basal removida ou perdida após a exploração.

\section{Dificuldades na modelagem de recrutamento}

Os dados disponíveis para os objetivos de modelagem são deficientes em pelo menos dois aspectos. Primeiro, por causa das técnicas tradicionais de medição de parcelas, a taxa de entrada de novos indivíduos geralmente tem sido baseada na taxa de ingresso em uma classe de tamanho de cerca de $10 \mathrm{~cm}$ de DAP ou maior e muito ocasionalmente encontram-se dados abaixo de $2 \mathrm{~cm}$. O problema de quando se usa taxa de ingresso em um diâmetro limite é o tempo de atraso. De fato, o ingresso atual depende parcialmente da regeneração de alguns anos anteriores. A segunda deficiência na disponibilidade dos dados é relativa à amplitude de condições de tipos florestais coberta pelos dados (LEAK; GRABER, 1976).

Um dos mais difíceis aspectos da modelagem de crescimento e produção florestal é a previsão da regeneração. Por causa da alta mortalidade entre plântulas, da dificuldade de identificação e do alto custo de medições, o inventário usualmente apenas avalia as árvores maiores (aquelas maiores de $10 \mathrm{~cm}$ de DAP), e o modelo de crescimento simula somente o crescimento dessa fração. É possível modelar o crescimento das árvores menores, porém os dados são escassos e isso adicionaria complexidade desnecessária, não fornecendo melhoria mensurável na precisão do modelo. Assim, normalmente os modelos predizem o recrutamento de árvores com $10 \mathrm{~cm}$ de DAP (VANCLAY, 1994a,b).

Os esforços para construir modelos que simulam o desenvolvimento de plantas, desde o estágio de semente, podem ser dificultados pela alta ocorrência de eventos aleatórios no desenvolvimento e germinação das sementes, pela falta de dados empíricos para ajustar o modelo, e pelo insuficiente entendimento dos processos fisiológicos envolvidos. Então, na prática, muitos modeladores recorrem aos modelos de regeneração e recrutamento. Como os dados adequados para modelagem da regeneração são freqüentemente difíceis de se obter, o recrutamento, em vez da regeneração, é o modelo mais freqüentemente usado para modelar a renovação da floresta (VANCLAY, 1994b).

De acordo com Vanclay (1992), uma das dificuldades na modelagem do recrutamento é a grande variabilidade na regeneração. Ao contrário do relativamente regular crescimento das árvores individuais, a regeneração tende a ser esporádica, com pouca ou nenhuma regeneração por vários anos $\mathrm{e}$ freqüentemente grandes quantidades nos anos em que ocorre. As condições do povoamento contribuem para algumas dessas variações, como a periodicidade de anos mais produtivos e o clima predominante, mas a regeneração permanece um processo estocástico, gerando dificuldades para uma eficiente estimativa do modelo. Boa parte da variabilidade associada com a regeneração é devida ao fato de que durante certo período alguma regeneração pode ou não ocorrer. Tais dados violam as suposições inerentes em análise de regressão e, se os dados estão repartidos em dois sistemas de estado, a habilidade de prever a quantidade de regeneração é bastante melhorada. Com um procedimento em dois estágios, a primeira equação estima a probabilidade com que alguma regeneração ou recrutamento irá ocorrer, e pode ser estimada usando uma regressão logística com presença/ausência como variável dependente. O segundo estágio é uma função condicional para predizer a quantidade de recrutamento, já considerando que o evento ocorrerá, e pode ser estimado usando uma regressão linear comum.

As estratégias de recrutamento são altamente variáveis em florestas pluviais, com diferenças interespecíficas no período de frutificação, número de sementes, tamanho de sementes, estratégia de dispersão, agentes, distâncias, sobrevivência da semente e probabilidade de germinação. Embora muitas vezes diferentes agentes de dispersão não ssejam diretamente distinguidos, a distância de dispersão média resultante depende da espécie (HUTH; KÖHLER, 2003).

Segundo Kohyama e Takada (1998), estimativas precisas das taxas de recrutamento e mortalidade requerem observações de mais árvores em parcelas de tamanho grande, ainda que isso carregue a desvantagem de aumentar os efeitos da heterogeneidade ambiental em espaço e aumente os custos de tais esforços de pesquisas. Estudos florestais podem usar a análise de tabelas do povoamento para calcular a probabilidade de transição em certo intervalo de tempo de uma determinada classe de tamanho para a próxima classe. Nesse procedimento, multiplica-se a densidade de árvores em certa classe pela taxa de crescimento. Cálculos similares podem ser usados para estimar as taxas de recrutamento de parcelas permanentes. Kohyama e Takada (1998) apresentaram a base teórica de um procedimento para estimar a taxa de recrutamento em um tamanho de classe mínimo em pequenas parcelas. 
Um outro problema é que as estimativas de taxas de recrutamento, e também de mortalidade, são afetadas por um fato potencialmente sério, que pode influenciar qualquer conclusão baseada em parcelas permanentes: as estimativas não são independentes do intervalo de tempo entre os inventários (LEWIS et al., 2004). Sheil e May (1996) mostraram que o mesmo efeito do intervalo entre medições que afeta a mortalidade é aplicado às taxas de recrutamento, quando ocorre um declínio nas taxas com o aumento do intervalo.

Porté e Bartelink (2002), em extensivo trabalho de revisão de modelos, afirmam que a modelagem do recrutamento é insatisfatória na maioria dos modelos, qualquer que seja o tipo. Diferenças entre valores observados e as estimativas são consideráveis: o modelo de árvore média de Lynch e Moser (1986), por exemplo, subestima em 35\% a 47\% o número de árvores recrutadas. Erros nas estimativas de recrutamento usando um modelo de árvore média, baseado em técnicas de regressão múltipla, resultam em erro de até $20 \%$ na estimativa final de volume em pé. As correlações entre o número de árvores recrutadas e a área basal do povoamento, o número de árvores e o DAP são usadas na maioria dos modelos, porém tais regressões resultam em modelos estatísticos pouco robustos. Modelos de clareiras também mostraram dificuldades na representação da regeneração. O modelo FORCAT, por exemplo, não é capaz de representar o número de árvores recrutadas por espécie de uma floresta mista.

Para modelos de clareiras, Porté e Bartelink (2002) citam um parâmetro específico que altera a estimativa do recrutamento: o tamanho da clareira. Uma clareira muito grande favorecerá as espécies intolerantes à sombra, enquanto que uma clareira muito pequena impedirá o estabelecimento, resultando em composição incorreta de espécies.

Shugart (1984) mostrou que a diversidade dos processos envolvidos na regeneração impediria o desenvolvimento de modelos mecanísticos. Entretanto, como afirmado por Liu e Ashton (1998), a representação da dispersão de sementes em modelos de dinâmica florestal é ainda mais necessária para compreender o recrutamento de espécies de árvores sem a presença de adultos no povoamento. Apesar da complexidade, tais modelos parecem muito promissores para representar a regeneração se forem acoplados a estudos intensivos sobre os diferentes processos, desde a produção de sementes até o recrutamento, para melhorar a acuracidade dos modelos.

A conseqüência da representação pouco robusta do recrutamento na modelagem do crescimento florestal foi pesquisada por vários autores. Em alguns casos, uma mudança de $25 \%$ nos parâmetros de recrutamento resulta em uma mudança de $17 \%$ a $18 \%$ na área basal do povoamento em uma simulação de 100 anos, bem como uma alteração da distribuição de diâmetros. Atenção especial deve ser dada à modelagem dos estágios iniciais. Tentativas para modelar o recrutamento com procedimentos mais mecanísticos foram feitos em diversos modelos de simulação de árvores dependentes de distância. Por exemplo, o modelo TROLL descreve a produção, dispersão e germinação de sementes para modelar o recrutamento. Como conseqüência, requer muitos parâmetros como a idade de maturação da árvore, a produção de sementes por árvore e a distância média de dispersão. O modelo de dinâmica florestal provou ser muito sensível aos valores desses parâmetros (PORTÉ; BARTELINK, 2002).

\section{Modelagem de recrutamento em modelos e sistemas}

Na maioria dos modelos derivados dos sistemas JABOWA/FORET, as plantas juvenis não são produzidas no modelo. Em vez disso, em cada iteração, as árvores recrutadas são obtidas de uma lista fixa de espécies. Esses modelos não consideram a dispersão nem a importante retroalimentação da dinâmica da população entre abundância e recrutamento. A seleção das árvores recrutadas pode ser totalmente aleatória ou pode ser modificada por fatores como o nível de herbivoria ou a disponibilidade de microssítios que favoreçam a germinação e o estabelecimento das plantas. Diversas das mais recentes versões desses modelos incluem tanto o recrutamento como a dispersão. Nesses modelos, algumas árvores recrutadas são obtidas a partir de uma lista fixa, enquanto em outros são produzidas pelas árvores no modelo e dispersas entre as células espaciais (PACALA et al., 1993).

O submodelo de recrutamento no modelo SORTIE prediz o sombreamento das plântulas de uma árvore individual, sendo a densidade de plântulas produzidas pela árvore uma função do tamanho e da distância da árvore. Foi desenvolvido então um método de máxima verossimilhança, que permite estimar um modelo de recrutamento para cada espécie a partir de um mapa de árvores adultas em um povoamento e de um censo de plântulas em parcelas localizadas no povoamento (PACALA et al., 1993). 
Ek e Monserud $(1974,1979)$ adotaram um procedimento mais sofisticado para predizer o recrutamento em seu modelo estocástico espacial para árvores (FOREST). O modelo de regeneração usou grupos representando o número de árvores para cada espécie e a idade em cada subparcela dentro da parcela principal. Apesar de haver registros de coleta de sementes em um período de 26 anos, os autores foram incapazes de ajustar um modelo para descrever a produção e usaram números aleatórios para selecionar boa, moderada e pobre produção anual de sementes, de acordo com a freqüência observada para cada espécie. A produção de sementes e de rebrotos foi estimada para cada árvore do estrato superior como uma função de seu tamanho e a idade limite, e foram distribuídos ao longo das subparcelas de acordo com a posição da árvore-mãe, a altura e a largura de copa. A germinação foi predita como uma função estocástica do microssítio e das condições de cobertura do dossel. A cada ano, uma germinante ou árvore no sub-bosque pode morrer, ou sobreviver e crescer em altura por uma quantidade predita a partir da cobertura, da espécie e da idade. Quando as árvores alcançam a altura do peito, elas são recrutadas dentro do modelo principal. Se as árvores não alcançam essa altura dentro de um tempo especificado, elas morrem.

Em estudo da dinâmica em floresta subtropical no sul do Brasil, realizado por Spathelf e Durlo (2001), o recrutamento foi avaliado registrando-se todas as árvores que atingiram o diâmetro limite de 5 cm durante o período 1994-1997. O processo de recrutamento foi modelado usando-se análise de regressão linear, na qual a área basal e o número de árvores dentro de certa classe de diâmetro foram considerados como possíveis parâmetros que afetam o recrutamento. Foi considerado que o recrutamento é afetado negativamente pela área basal e positivamente pelo número total de árvores por unidade de área.

No modelo FORMIND (HUTH; KÖHLER, 2003), dois diferentes mecanismos de recrutamento foram a ele incorporados. O procedimento mais simples (chamado CONSTANT) considera que uma floresta intacta tem uma taxa constante de entrada de sementes, o que é um procedimento adequado para florestas não-perturbadas. O segundo procedimento (chamado LOCAL) leva em conta a dispersão de sementes produzida pelas árvores-mães locais, isto é, árvores que excedem certo tamanho. Este segundo procedimento é necessário para suposições sobre a falta de recrutamento. Sete cenários com diferentes reduções de taxas de recrutamento foram definidos, desde uma floresta com produção constante de sementes (caso referência) até uma floresta sem qualquer recrutamento. $O$ último cenário pode ser muito improvável, porém ele possibilita a análise da dinâmica do modelo independente do módulo de recrutamento.

Kohyama et al. (2003), em estudo de floresta mista de dipterocarpáceas na Indonésia, observaram que o número de árvores recrutadas era limitado e, inevitavelmente, subestimado, porque não era possível registrar o número de árvores recrutadas e que morreram. Assim, foi aplicada a técnica da estimativa $G f$ (KOHYAMA; TAKADA, 1998). A estimativa $G f$ para o centro de classe de tamanho mínimo examinada é a densidade $f$ (número de árvores $\mathrm{ha}^{-1}$ ) na classe de tamanho mínima (no caso, 5-7 cm de DAP), multiplicada pela taxa de crescimento absoluta média $G$ ( $\left.\mathrm{cm}^{2} \mathrm{ano}^{-1}\right)$ dos sobreviventes dentro da classe, dividido pelo tamanho da classe $(2 \mathrm{~cm})$. A eficiência da taxa de recrutamento é definida como $R$, dividido por uma medida de abundância para as espécies, e para isso seis variáveis foram testadas: densidade da árvore; área basal; $\mathrm{DAP}^{3}$ cumulativo; $\mathrm{DAP}^{5}$ cumulativo; massa foliar; e taxa de crescimento vegetativo (biomassa), todas definidas para árvores com DAP $\geq 6 \mathrm{~cm}$. A melhor variável foi o DAP cumulativo, com coeficiente de determinação $\left(\mathrm{R}^{2}\right)$ de 0,93 .

O modelo NORM (VANCLAY, 1992), utilizado na Austrália, usa um procedimento de dois estágios para predizer a regeneração que ocorre para cada uma das 100 espécies que contribuem com 97 $\%$ de todo recrutamento (a $10 \mathrm{~cm}$ de DAP) registrado em parcelas permanentes. Os 3\% remanescentes compreendem 113 espécies e contribuem com dados insuficientes para análises significativas de características da regeneração. Não há maneira fácil para decidir quantas espécies utilizar para modelar o recrutamento. Nas florestas pluviais de Queensland, 60 espécies contribuíram com 90\% de todo o recrutamento, 80 espécies com 95\% e 100 espécies com 97\% do total observado. O número ótimo de espécies para modelar depende muito dos dados e recursos disponíveis, sobretudo do uso pretendido do modelo (VANCLAY, 1994b).

Todos os modelos de distribuição independentes da distância tratam o recrutamento em nível de povoamento, isto é, como o número de pequenas árvores que surgem durante um período de tempo por unidade de área. O recrutamento tem sido modelado usando-se tanto um valor constante por hectare (modelo HOPSY) quanto como uma relação positiva ou negativa com a área basal do povoamento ou 
densidade do povoamento (FOREST, FIBER 3.0). As equações de regressões usadas diferiram dependendo da disponibilidade de dados, sendo a maioria linear (FIBER 3.0, CAFOGROM), ou descritos com equação de regressão exponencial (FOREST, SIRENA). O diâmetro das árvores que ingressam foi sempre determinado empiricamente, sendo estabelecida qual a média observada do DAP de novas árvores por espécie (CAFOGROM) ou para todas as espécies (PORTÉ; BARTELINK, 2002).

Em modelo para árvores dependentes da distância, Porté e Bartelink (2002) afirmam que o recrutamento deve ser modelado não somente pelo número e dimensões das plantas, mas também pela sua localização. Nos primeiros modelos, um procedimento elementar foi escolhido, não modelando a produção de sementes e dispersão por si só, enquanto que os modelos mais recentes tendem a ser mais mecanísticos. A localização das plântulas é então selecionada ao acaso entre as áreas abertas do povoamento. O procedimento usado na maioria dos sistemas corresponde ao tipo de recrutamento apresentado em muitos modelos de clareiras, com a diferença de que o recrutamento é estimado em nível de árvores (FOREST, SORTIE, ARCADIA, FORMOSAIC, TROLL). Em todos esses modelos, a dispersão de sementes sempre requer a presença de árvores adultas próximas. No modelo FORMOSAIC, foi desenvolvido um procedimento original multiescalar, em que a floresta-alvo é integrada em uma paisagem de áreas florestadas e não-florestadas. O recrutamento em uma célula resulta não somente da presença de árvores adultas dentro da floresta, mas também da dispersão de sementes à longa distância das árvores adultas localizadas nos arredores da floresta modelada. Como uma conseqüência, as características das áreas adjacentes podem influenciar o número e a espécie das árvores recrutadas.

De acordo com Porté e Bartelink (2002), em modelos de não-clareiras para árvores, independentes da distância, o recrutamento é geralmente modelado em nível de povoamento. O número de árvores recrutadas por espécie é representado como uma função de variáveis do povoamento tais como área basal, densidade e proporção de espécies (BOJRES, PROGNAUS) e também características do sítio, como profundidade do solo e altitude (FIRE-BCG, PROGNAUS). A dispersão das sementes foi incluída no modelo FIRE-BCG como cenário para modelar os efeitos específicos do fogo na ocorrência da regeneração.

Porté e Baterlink (2002), em uma revisão de modelos para florestas mistas, afirmam que o recrutamento, em modelos de clareiras independentes da distância, é modelado como um processo estocástico ativado pela disponibilidade de luz e, ocasionalmente, por outras condições ambientais. Uma espécie, para se estabelecer na parcela, dependerá das condições ambientais, bem como das necessidades ecológicas da espécie, como a intensidade mínima de luz no nível do solo e condições de umidade do solo (modelos JABOWA, FORET, KIAMBRAM, FORCAT, FORSKA, OUTENIQUA, FORECE, MIOMBO e KOPIDE). Algumas vezes são adicionados outros critérios, como a presença ou ausência de animais (FORET), as necessidades nutricionais relacionadas ao solo mineral ou liteira (FORET, FORCAT) e a presença de pássaros ou ocorrência de ventos que assegurem a dispersão (KIMBRAM, OUTENIQUA). O número de plantas juvenis produzido por cada espécie elegível é determinado aleatoriamente, dentro de um certo limite (JABOWA, FORET, KIAMBRAM, FORCAT, FORSKA, OUTENIQUA, MIOMBO). Um modo alternativo de modelar o número de árvores jovens consiste em ajustar o número delas para um valor máximo por espécie, número este que irá então diminuir devido às condições ambientais indesejáveis, tais como baixa disponibilidade de luz, baixas temperaturas e baixa umidade do solo, ou usando as funções limitantes (ZELIG, FORGRA), ou escolhendo uma taxa de sobrevivência aleatória (KOPIDE). O modelo FORCAT é o único modelo de clareira que apresenta modos de recrutamento mais mecanístico, pois a disponibilidade de sementes em uma clareira depende da produção de sementes dentro da clareira e da dispersão de sementes originárias de fora da clareira, usando um limite de probabilidade à presença prévia de uma árvore adulta próxima e a probabilidade de sementes entrarem na clareira, que é negativamente dependente do peso da semente. Nos modelos, o tamanho das plantas é algumas vezes uniforme, por exemplo, FORECE e FORCLIM, nos quais as plantas das mesmas espécies entram na clareira com exatamente o mesmo DAP, constituindo um grupo. Em outros modelos de clareira, os DAP (e/ou altura das plantas) são selecionados aleatoriamente, a partir de uma amplitude pré-definida (JABOWA, FORET, OUTENIQUA, FORSKA, MIOMBO). De acordo com a floresta e as espécies, algumas vezes a brotação é considerada uma estratégia alternativa de regeneração (FORET, FORCAT, FORECE). As espécies elegíveis, o número e as dimensões dos brotos são então estimados de um modo similar ao do recrutamento de árvores. 


\section{Taxas de recrutamento em florestas}

Em uma análise mundial da dinâmica de florestas tropicais, Phillips et al. (1994) estudaram 25 sítios em quatro continentes. As taxas de recrutamento variaram de $0,397 \%$ a $2,827 \%$. Os menores valores foram obtidos em Queensland (Austrália) e Belém (Pará), e os maiores na Amazônia peruana e na Costa Rica. Os autores desenvolveram um modelo logarítmico para estimar o recrutamento anual na classe de DAP de $10 \mathrm{~cm}$.

A taxa de recrutamento encontrada por Swaine et al. (1987) em uma floresta úmida semidecídua em Gana foi em média de 1,56\% ao ano, em um período de observação de 14 anos. Valores médios semelhantes foram obtidos por Manokaran e Kochummen (1987) em uma floresta de dipterocarpáceas na Malásia, onde os valores variaram entre 1,1\% a 2,2\% ao ano, em um período de observação de 34 anos.

Castro Marín et al. (2005) encontraram taxa de recrutamento média em floresta tropical seca na Nicarágua de 2,5\% ao ano, no período 1993-2000, para DAP $\geq 10 \mathrm{~cm}$. Para DAP $\geq 2,5 \mathrm{~cm}$, Lang e Knight (1983) obtiveram taxa de recrutamento de 0,9\% ao ano em Barro Colorado, no Panamá, em um período de dez anos, sendo que o recrutamento foi maior nas áreas de clareiras.

Condit et al. (1993), estudando a dinâmica da espécie Prioria copaifera no Panamá, obtiveram valor anual de recrutamento de 0,47 árvore para cada árvore adulta. $\mathrm{O}$ valor do recrutamento foi próximo ao dobro do número total de mortes em cada intervalo do censo, e o total do recrutamento excedeu o número combinado de árvores que morreram ou quebraram. A maioria das árvores recrutadas media menos de $3 \mathrm{~cm}$ de DAP.

Silva et al. (1995), em floresta explorada no estado do Pará, detectaram que após a exploração o ingresso de novas plantas na classe de DAP $\geq 5 \mathrm{~cm}$ aumentou substancialmente durante os oito primeiros anos $(5,2 \%)$. Durante os outros cinco anos houve uma queda acentuada, tanto em termos relativos como absolutos para todas as espécies $(1,8 \%)$, bem como para o grupo de espécies comerciais (1,3\%). Alguns anos após a exploração, as condições de luz no sub-bosque da floresta não mais promovem o rápido crescimento de plântulas e mudas. A taxa média de recrutamento anual foi de 3,1\%, para o período de 13 anos de observações após a exploração.

O recrutamento encontrado em floresta de cerrado próxima a Brasília foi de 2,67\% ao ano, para um período de seis anos, e foi menor do que a mortalidade (3,5\%). A relação entre recrutamento e densidade foi significante, apresentando coeficiente de correlação $(r)$ de 0,72. Embora também significante, a correlação entre recrutamento e área basal das espécies foi mais fraca $(r=0,24)$ que a correlação entre mortalidade e área basal (FELFILI, 1993, 1995). A forte correlação positiva entre mortalidade, recrutamento e densidade de espécies sugere que o alto recrutamento das espécies abundantes tende a compensar a mortalidade mais alta, assim conservando a dominância (FELFILI, 1995).

Scolforo et al. (1996), em uma Floresta Montana Semidecídua no estado de Minas Gerais, encontrou ingresso aos $5 \mathrm{~cm}$ de DAP de 5,5\% ao ano em observação de cinco anos. Para floresta também semidecídua na Mata Atlântica (ES), considerando todas as espécies, o recrutamento encontrado por Rolim et al. (1999) foi de 1,95\% ao ano, chegando a 3\% para as espécies pioneiras.

Em observações feitas em floresta tropical atlântica na Ilha do Cardoso (SP), Melo (2000) encontrou taxa anual de recrutamento de 2,43\% para dominantes (DAP $>9,9 \mathrm{~cm}$ ) e 2,3\% para espécies do sub-bosque (DAP $>2,5 \mathrm{~cm}$ ), em 6,8 anos de coleta de dados. Excluindo-se as palmeiras, a taxa reduziu para $1,45 \%$ e 2,08\%, respectivamente. Comparando florestas com araucária no estado do Paraná, Sanquetta et al. (2003) encontraram valores de recrutamento de 5,58\% em quatro anos para todas as espécies em uma floresta e $2,87 \%$ em oito anos para outra floresta, e em ambos os casos a taxa foi superior à mortalidade.

Schaaf (2001), estudando uma floresta ombrófila mista no sul do estado do Paraná, encontrou taxa anual média de recrutamento de 1,3\%, entre dois inventários realizados com intervalo de 21 anos, considerando árvores com DAP $\geq 20 \mathrm{~cm}$. Spathelf e Durlo (2001), estudando a dinâmica de floresta subtropical úmida no estado do Rio Grande do Sul, obtiveram recrutamento de 9,5\% em três anos de observações. Esses dados indicam que a floresta sob estudo está, mesmo em partes, em um processo de renovação. $\mathrm{O}$ recrutamento foi avaliado registrando-se todas as árvores que ultrapassaram o diâmetro limite de $5 \mathrm{~cm}$. 


\section{CONSIDERAÇÕES FINAIS}

O recrutamento apresenta uma grande variabilidade espacial e temporal, fato este que dificulta a modelagem desse evento em florestas. Além disso, existe a dificuldade de obtenção de dados para populações de plantas com DAP inferior a $10 \mathrm{~cm}$, o qual parece ser um limite adotado que combina menor custo e melhor acuidade para ajuste dos modelos. Nesse caso, a modelagem abrange somente as árvores maiores, não incluindo as fases de plântulas, mudas e arvoretas. Isso define os modelos de recrutamento e os de regeneração.

Alguns autores recomendam a modelagem em dois estágios, utilizando-se uma função do tipo logística para modelar a probabilidade de ocorrência do recrutamento e uma segunda função condicional (normalmente linear) para modelar o número de árvores recrutadas, caso ocorra o evento.

As variáveis mais correlacionadas com o recrutamento são a área basal do povoamento ou do grupo de espécies, a densidade do povoamento e o índice de competição. O mais adequado é o uso de funções fisiológicas e/ou ecológicas, porém isto demanda uma quantidade muito maior de informações, as quais não estão disponíveis para florestas, impedindo ou dificultando o uso de modelos mecanísticos.

\section{REFERÊNCIAS}

ALDER, D.; SILVA, J. N. M. An empirical cohort model for management of Terra Firme forests in the Brazilian Amazon. Forest Ecology and Management, Amsterdam, v. 130, n. 1-3, p. 141-157, 2000.

BOTKIN, D. B., JANAK, J. F.; WALLIS, J. R. Some ecological consequences of a computer model of forest growth. Journal of Ecology, Oxford, v. 60, n. 3, p. 849-872, 1972.

CARVALHO, J. O. P. Dinâmica de florestas naturais e sua implicação para o manejo florestal. In: CURSO DE MANEJO FLORESTAL SUSTENTÁVEL. Tópicos em manejo florestal sustentável. Colombo: Embrapa-CNPF, 1997. p. 43-55.

CASTRO MARÍN, G.; NYGARD, R.; GONZALES RIVAS, B.; ODEN, P. C. Stand dynamics and basal area change in a tropical dry forest reserve in Nicaragua. Forest Ecology and Management, Amsterdam, v. 208, n. 1-3, p. 63-75, 2005.

CLARK, J. S.; BECKAGE, B.; CAMILL, P.; CLEVELAND, B.; LAMBERS, J. H. R.; LICHTER, J.; MCLACHLAN, J.; MOHAN, J.; WYCKOFF, P. Interpreting recruitment limitation in forests. American Journal of Botany, Columbus, v. 86, n. 1, p. 1-16, 1999.

CONDIT, R.; HUBBELL, S. P.; FOSTER, R. B. Mortality and growth of a commercial hardwood 'el cativo', Prioria copaifera, in Panama. Forest Ecology and Management, Amsterdam, v. 62, n. 1-4, p. 107-122, 1993.

EK, A. R.; MONSERUD, R. A. Trials with program FOREST: growth and reproduction simulation for mixed species even- or uneven-aged forest stands. In: IUFRO WORKING PARTY S4.01-4 MEETINGS, 1973, Growth models for tree and stand simulation. Stockholm: Dep. For. Yield res., Royal College Forestry, 1974. p. 56-69.

EK, A. R.; MONSERUD, R. A. Performance and comparison of stand growth models based on individual tree and diameter-class growth. Canadian Journal of Forest Research, Ottawa, v. 9, p. 231-244, 1979.

FELFILI, J. M. Growth, recruitment and mortality in the Gama gallery forest in central Brazil over a sixyear period (1985-1991). Journal of Tropical Ecology, Cambridge, v. 11, n. 1, p. 67-83, 1995.

FELFILI, J. M. Structure and dynamics of a gallery forest in central Brazil. $180 \mathrm{f}$. Thesis (Doctor of Philosophy) - Oxford Forestry Institute / Department of Plant Sciences, University of Oxford, Oxford, 1993.

HANN, D. W. Development and evaluation of an even- and uneven-aged ponderosa pine/Arizona Fescue stand simulator. Ogden: Forest Service/Intermountain Forest and Range Experiment Station, 1980. 95 p. (Research Paper, n. 267) 
HUEBSCHMANN, M. M.; GERING, L. R.; LYNCH, T. B.; BITOKI, O.; MURPHY, P. A. An individual-tree growth and yield prediction system for uneven-aged shortleaf pine stands. Southern Journal of Applied Forestry, Bethesda, v. 24, n. 2, p. 112-120, 2000.

HUTH, A.; KÖHLER, P. Impacts of logging in tropical rain forests with disturbed recruitment - a simulation study. In: DECISION SUPPORT FOR MULTIPLE PURPOSE FORESTRY. A TRANSDISCIPLINARY CONFERENCE ON THE DEVELOPMENT AND APPLICATION OF DECISION SUPPORT TOOLS FOR FOREST MANAGEMENT., 2003, Vienna. Conference Proceedings... Vienna: University of Natural Resources and Applied Life Sciences, 2003. p. 1-10.

KOHYAMA, T. Size-structured multi-species model of rain forest trees. Functional Ecology, Oxford, v. 6, n. 2, p. 206-212, 1992.

KOHYAMA, T.; SUZUKI, E.; PARTOMIHARDJO, T.; YAMADA, T. Dynamic steady state of patchmosaic tree-size structure of a mixed dipterocarp forest regulated by local crowding. Ecological Research, Tsukuba, v. 16, n.1, p. 85-98, 2001.

KOHYAMA, T.; SUZUKI, E.; PARTOMIHARDJO, T.; YAMADA, T.; KUBO, T. Tree species differentiation in growth, recruitment and allometry in relation to maximum height in a Bornean mixed dipterocarp forest. Journal of Ecology, Oxford, v. 91, n. 5, p. 797-806, 2003.

KOHYAMA, T.; TAKADA, T. Recruitment rates in forest plots: Gf estimates using growth rates and size distributions. Journal of Ecology, Oxford, v. 86, n. 4, p. 633-639, 1998.

LANG, G. E.; KNIGHT, D. H. Tree growth. mortality, recruitment, and canopy gap formation during a 10-year period in a tropical moist forest. Ecology, Tempe, v. 64, n. 5, p. 1075-1080, 1983.

LEAK, W. B.; GRABER, R. E. Seedling input, death, and growth in uneven-aged northern hardwoods. Canadian Journal of Forest Research, Ottawa, v. 6, n. 3, p. 368-374, 1976.

LEWIS, S. L.; PHILLIPS, O. L.; SHEIL, D.; VINCETI, B.; BAKER, T. R.; BROWN, S.; GRAHAM, A. W.; HIGUCHI, N.; HILBERT, D. W.; LAURANCE, W. F.; LEJOLY, J.; MALHI, Y.; MONTEAUDO, A.; NÚÑEZ VARGAS, P.; SONKÉ, B.; SUPARDI, N.; TERBORGH, J. W.; VÁSQUEZ MARTÍNEZ, R. Tropical forest tree mortality, recruitment and turnover rates: calculation, interpretation and comparison when census intervals vary. Journal of Ecology, Oxford, v. 92, n. 6, p. 929-944, 2004.

LEXERØD, N.; EID, T. Recruitment models for Norway spruce, Scots pine, birch and other broadleaves in young growth forests in Norway. Silva Fennica, Helsinki, v. 39, n. 3, p. 391-406, 2005.

LIU, J. G.; ASHTON, P. S. FORMOSAIC: an individual-based spatially explicit model for simulating forest dynamics in landscape mosaics. Ecological Modelling, Amsterdam, v. 106, n. 2-3, p. 177-200, 1998.

LYNCH, T. B.; MOSER JR., J. W. A growth model for mixed species stands. Forest Science, Bethesda, V. 32, n. 3, p. 697-706, 1986.

MANOKARAN, N.; KOCHUMMEN, K. M. Recruitment, growth and mortality of tree species in a lowland dipterocarp forest in Peninsular Malaysia. Journal of Tropical Ecology, Cambridge, v. 3, n. 4, p. 315-330, 1987.

MELO, M. M. R. F. Demografia de árvores em floresta pluvial tropical atlântica, Ilha do Cardoso, SP, Brasil. 155 f. Tese (Doutorado em Ecologia) - Instituto de Biociências, Universidade de São Paulo, São Paulo, 2000.

MOSER JR., J. W. Dynamics of an uneven-aged forest stand. Forest Science, Bethesda, v. 18, n. 3, p. 184-191, 1972.

MUHAIRWE, C. K. Biometric Models. Sydney: Resource and Conservation Assessment Council, 2003. 46 p. (NAND 08; v. 5.). 
NAMAALWA, J.; EID, T.; SANKHAYAN, P. A multi-species density-dependent matrix growth model for the dry woodlands of Uganda. Forest Ecology and Management, Amsterdam, v. 213, n. 1-3, p. 312$327,2005$.

PACALA, S. W.; CANHAM, C. D.; SILANDER Jr., J. A. Forest models defined by field measurements: I. The design of a northeastern forest simulator. Canadian Journal of Forest Research, Ottawa, v. 23, n. 10, p. 1980-1988, 1993.

PHILLIPS, O. L.; HALL, P.; GENTRY, A. H.; SAWYER, S. A.; VÁSQUEZ, R. Dynamics and species richness of tropical rain forests. Proceedings of the National Academy of Sciences of United States of America, Washington, DC, v. 91, n. 7, p. 2805-2809, 1994.

PORTÉ, A.; BARTELINK, H. H. Modelling mixed forest growth: a review of models for forest management. Ecological Modelling, Amsterdam, v. 150, p. 141-188, 2002.

ROBERT, A. Simulation of the effect of topography and tree falls on stand dynamics and stand structure tropical forests. Ecological Modelling, Amsterdam, v. 167, n. 3, p. 287-303, 2003.

ROLIM, S. G.; COUTO, H. T. Z.; JESUS, R. M. Mortalidade e recrutamento de árvores na Floresta Atlântica em Linhares (ES). Scientia Forestalis, Piracicaba, n. 55, p. 49-69, 1999.

SANQUETTA, C. R.; CÔRTE, A. P. D.; EISFELD, R. L. Crescimento, mortalidade e recrutamento em duas florestas de Araucária (Araucaria angustifolia (Bert.) O. Ktze.) no estado do Paraná, Brasil. Revista Ciências Exatas e Naturais, Guarapuva, v. 5, n. 1, p. 101-112, 2003.

SCHAAF, L. B. Florística, estrutura e dinâmica no período 1979-2000 de uma floresta ombrófila mista localizada no sul do Paraná. 119 f. Dissertação (Mestrado em Engenharia Florestal) - Setor de Ciências Agrárias, Universidade Federal do Paraná, Curitiba, 2001.

SCOLFORO, J. R. S.; PULZ, F. A.; MELLO, J. M.; OLIVEIRA FILHO, A. T. Modelo de produção para floresta nativa como base para manejo sustentado. Cerne, Lavras, MG, v. 2, n. 1, p. 112-137, 1996.

SHEIL, D.; MAY, R. M. Mortality and recruitment rate evaluations in heterogeneous tropical forests. Journal of Ecology, Oxford, v. 84, n. 1, p. 91-100, 1996.

SHUGART, H. H. A theory of forest dynamics: the ecological implications of forest succession models. New York: Springer-Verlag, 1984. 278 p.

Silva, J. N. M.; CARVAlho, J. O. P.; LOPES, J. C. A.; AlMEIDA, B. F.; COSTA, D. H. M.; OLIVEIRA, L. C.; VANCLAY, J. K.; SKOVSGAARD, J. P. Growth and yield of a tropical rain forest in the brazilian Amazon after 13 years after logging. Forest Ecology and Management, Amsterdam, v. 71, n. 3, p. 267-274, 1995.

SPATHELF, P.; DURLO, M. A. Transition matrix for modeling the dynamics of a subtropical seminatural forest in southern Brazil. Forest Ecology and Management, Amsterdam, v. 151, n. 1-3, p. 139-149, 2001.

SWAINE, M. D.; HALL, J. B.; ALEXANDER, I. J. Tree population dynamics at Kade, Ghana (19681982). Journal of Tropical Ecology, Cambridge, v. 3, n. 4, p. 331-345, 1987.

VANCLAY, J. K. A growth model for North Queensland rainforests. Forest Ecology and Management, Amsterdam, v. 27, n. 3-4, p. 245-271, 1989.

VANCLAY, J. K. Modelling regeneration and recruitment in a tropical rain forest. Canadian Journal of Forest Research, Ottawa, v. 22, n. 9, p. 1235-1248, 1992.

VANCLAY, J. K. Sustainable timber harvesting: simulation studies in the tropical rain forests of north Queensland. Forest Ecology and Management, Amsterdam, v. 69, n. 1-3, p. 299-320, 1994a.

VANCLAY, J. K. Modelling forest growth and yield. Wallingford: CAB International, 1994b. 312 p. 